\title{
TESTING PROTON SPIN MODELS WITH POLARIZED BEAMS *
}

\author{
Gordon P. Ramsey \\ Physics Department, Loyola University, Chicago, IL 60626 \\ and \\ High Energy Physics Division, Argonne National Laboratory, Argonne, IL 60439
}

\begin{abstract}
We review models for spin-weighted parton distributions in a proton. Sum rules involving the nonsinglet components of the structure function $x g_{1}^{p}$ help varrow the range of parameters in these models. The contribution of the $\gamma_{5}$ anomaly term depends on the size of the integrated polarized gluon distribution and experimental predictions depend on its size. We have proposed three models for the polarized gluon distributions, whose range is considerable. These model distributions give an overall range of parameters that can be tested with polarized beam experiments. These are discussed with regard to specific predictions for polarized beam experiments at energies typical of UNK.
\end{abstract}

\section{DISCLAIMER}

\begin{abstract}
This report was prepared is an account of work sponsored by an agency of the United States Government. Neither the Uniied States Government nor any agency thereof, nor any of their employees, makes any warranty, express or implied, or assumes any legal liability or responsibility for the accuracy, completeness, or usefulness of any information, apparatus, product, or process disclosed, or represents that its use would not infringe privately owned rights. Reference herein : $:$ any specific commercial product, process, or service by trade name, trademark, manufacturer, or otherwise does not necessarily constitute or imply its endorsement, recommendation, or favoring by the United States Government or any agency thereof. The views and opinions of authors expressed herein do not necessarily state or reflect those of the United States Government or any agency thereof.
\end{abstract}

* Presented at the IV Workshop on High Energy Spin Physics; Protvino, Moscow Region, USSR; September 3, 1991. Work supported by the U.S. Department of Energy, Division of High Energy Physics, Contract 
Measuring spin-observables with polarized proton beams provides an excellent opportunity to enhance our knowledge of QCD. Several papers ${ }^{1,2,3}$ have provided phenomenological estimates for spin-weighted inclusive scattering processes based on simple models for the parton spin densities. Recent technical developments involving polarized beams and targets will now allow hard-scattering processes to be measured in kinematic regimes where QCD perturbation theory can be valid. ${ }^{4}$ The results from the EVIC ${ }^{5}$ suggest a strong negative polarization for the sea of $q \bar{q}$ pairs and have created controversy regarding reconciliation of the data with the parton model predictions. This stresses the importance of understanding how the spin of the proton is shared among the spin of its constituents and orbital angular momentum. It is crucial to assign a high priority to a comprehensive experimental program involving polarized beams and targets aimed at answering the many questions that still exist. In planning polarized beam and target experiments, it is useful to have models for the spin transfer densities which will allow experimenters to assess the precision required to extract the necessary information. We have constructed simple model distributions for the polarization of the valence quarks, the gluons and the sea using existing data on the longitudinal spin-spin asymmetry in deep-inelastic lepton-proton scattering and the sum rules involving the polarized parton distributions.

In our models, we distinguish between the "valence" and the "sea" quarks of the proton to construct the spin-weighted distributions. The polarized valence quark distributions are based on a broken SU(6) model, written in terms of the unpolarized distributions with a flavor independent factor, which is connected to a possible nonperturbative polarization of the sea and glue. These valence distributions have been discussed elsewhere in detail. ${ }^{1}$ Coupled with the Bjorken sum rule, this model implies that the valence quarks carry about $77 \%$ of the proton's spin, consistent with a Skyrme model approach. ${ }^{6}$

Recent attention to the $\gamma_{5}$ anomaly $^{7}$ has allowed speculation that the polarized gluon density can affect the relationship between the "observable" quark and antiquark spin densities and those densities which have simple chiral properties in the limit $m_{q} \rightarrow 0$. Traditionally, this distribution was thought to be limited by the gluon's momentum. However, analysis of the EMC data indicate that it may be significantly larger. Since the QCD evolution equations $^{8}$ do not specify the size of $\Delta G\left(Q^{2}\right)$ at any particular $Q^{2}$, it is important to attempt to measure $\Delta G\left(x, Q^{2}\right)$ in some processes. Possible models for $\Delta G$ fall into three basic categories. First, we can assume $\Delta G=0$ for all $x$ at some value of $Q_{0}^{2}$. The polarization 
of the gluons is generated using the spin-weighted QCD evolution equations. This model is consistent with the Skyrme approach to the proton structure. A second model assumes $\Delta G \simeq x G$ at $Q_{0}^{2} \simeq 10.7 \mathrm{GeV}^{2}$, so that the net spin carried by gluons is approximately equal to the fraction of momentum that they carry: $\left\langle\Delta G\left(Q_{0}^{2}\right)\right\rangle \equiv \int_{0}^{1} d x \cdot x G\left(x, Q^{2}\right) \simeq 0.50$. Finally, one can assume that the spin-spin forces lead to a saturation of the gluon polarization and $\Delta G \simeq G$ for $x \geq x_{0}$, for some chosen $x_{0}$. This possibility is suggested by the discrepancy of the EMC data with certain theoretical estimates on the amount of sea polarization allowed. If we assume that the gluon density follows the second model for $x_{0} \leq 0.04$ and saturates thereafter, then $\langle\Delta G(x)\rangle \simeq 5.0$ This last model gives considerable difference between the measured and calculated values of the spin fraction of the proton carried by quarks.

Data suggest that the sea pairs are polarized opposite to the proton's spin. ${ }^{5}$ However, there is still considerable uncertainty concerning the magnitude of the sea polarization. For the sea distribution, we assume that there is an enhancement of up and down quarks/antiquarks over strange quarks/antiquarks due to the difficulty of polarizing heavier flavors, and that spin-spin correlation forces tend to enhance the polarization of antiquarks over quarks in the sea. Thus, we make the distinction ialat

$$
\Delta \bar{q}\left(x, Q_{0}^{2}\right)=c(x) \Delta q_{s}\left(x, Q_{0}^{2}\right)
$$

at some $Q_{0}^{2}$ for each flavor. The parameter $c(x)$ represents the enhancement of polarized antiquarks over polarized quarks in the sea. Additionally, we assume isospin invariance of the sea with respect to the up and down quarks, but a broken flavor $\mathrm{SU}(3)$ sea so that

$$
\begin{aligned}
\Delta u_{s} & =\Delta d_{s}=[1+\epsilon(x)] \Delta s \\
\Delta \bar{u} & =\Delta \bar{d}=[1+\bar{\epsilon}(x)] \Delta \bar{s} .
\end{aligned}
$$

The symmetry breaking parameter $\epsilon$ indicates the difficulty in polarizing the strange quarks due to their larger masses. Thus,

$$
\Delta \bar{u}=c \Delta u_{s}=\Delta \bar{d}=c \Delta d_{s}=(1+\bar{\epsilon}) \Delta \bar{s}=c(1+\epsilon) \Delta s
$$

One sea model uses the EMC experimental determination of $\int_{0}^{1} d x \cdot g_{1}^{p}\left(x, Q^{2}\right)$ to determine the sea distributions explicitly. This and $A_{8}$ sum rule can be used to determine the polarized 
sea parameters. Then, the total spin carried by each of the quark flavors including valence and sea is

$$
\begin{gathered}
\left\langle\Delta u_{v}\right\rangle+\left\langle\Delta u_{s}+\Delta \bar{u}\right\rangle=1.02+(-0.24)=0.78 \\
\left\langle\Delta d_{v}\right\rangle+\left\langle\Delta d_{s}+\Delta \bar{d}\right\rangle=-0.25+(-0.24)=-0.49 \\
\langle\Delta s+\Delta \bar{s}\rangle=-0.15
\end{gathered}
$$

The total contribution from all flavors of sea quarks in this model is $-0.63 \pm 0.60$. The experimental errors of determining the parameters in the sum rules are reflected in this number. If we assume that the polarized gluon distribution is large, say $\langle\Delta G\rangle \simeq 5$, then when the above results are translated into parton model language, we find that the total spin carried by all quarks is about 0.79 to within experimental errors, consistent with the naive quark model. A theoretical concern with this model is that the spin carried by the sea quark pairs is much larger than the corresponding momentum. In an alternate model, the spin transfer densities of the sea anti-quarks and quarks are assumed to be limited. This implies a large value of $\epsilon$, indicating that the strange sea is much harder to polarize, due to mass differences of the quark flavors. For a small polarized gluon distribution, the fraction of spin carried by all quarks in this model is about one half of the proton spin, which is significantly greater than that implied by the EMC motivated model. Here, the spin carried by the quark flavors is

$$
\begin{gathered}
\left\langle\Delta u_{v}\right\rangle+\left\langle\Delta u_{s}+\Delta \bar{u}\right\rangle=1.02+(-0.10)=0.92 \\
\left\langle\Delta d_{v}\right\rangle+\left\langle\Delta d_{s}+\Delta \bar{d}\right\rangle=-0.25+(-0.10)=-0.35 \\
\langle\Delta s+\Delta \bar{s}\rangle=-0.01
\end{gathered}
$$

Only a comprehensive experimental program in polarized beams can help to distinguish the accuracy of these theoretical models. The valence quark distributions can be determined from existing data on the longitudinal asymmetry in deep-inelastic lepton-proton scattering. Further precision measurements of the lepton-hadron scattering asymmetry are needed at small- $x$ to reduce the uncertainties of the polarized valence quark distributions. The accelerators at CERN and Hamburg (HERA) can probe smaller $x$ for both proton and neutron targets. A natural extension of the work on the proton involves a measurement of $A_{1}^{\text {en }}$, which would allow an experimental test of the Bjorken sum rule. ${ }^{9}$ Some ${ }^{10}$ have indicated that our understanding of QCD may be flawed in such a way to allow a violation of the Bjorken 
sum rule. Measurement of $A_{1}^{\text {en }}$ would provide significant additional information concerning nucleon spin structure.

The Drell-Yan process allows the most direct probe of the polarization of the antiquarks in the sea. ${ }^{11}$ After integrating over transverse momentum, the spin-spin asymmetry for the production of lepton pairs in $p p$ collisions can be given by

$$
\frac{a_{L L} d \sigma}{d M^{2} d x_{F}}=\frac{4 \pi \alpha^{2}}{3 M^{2}} \cdot \frac{1}{3} \cdot \sum_{q} e_{q}^{2}\left[x_{F}^{2}+\frac{4 M^{2}}{s}\right]^{-\frac{1}{2}}\left[\Delta q\left(x_{a}\right) \Delta \bar{q}\left(x_{b}\right)+\Delta \bar{q}\left(x_{a}\right) \Delta q\left(x_{b}\right)\right]
$$

where $x_{a}=\frac{1}{2}\left[\left(x_{F}^{2}+4 \frac{M^{2}}{s}\right)^{\frac{1}{2}}+x_{F}\right]$ and $x_{b}=\frac{1}{2}\left[\left(x_{F}^{2}+4 \frac{M^{2}}{s}\right)^{\frac{1}{2}}-x_{F}\right]$. To the extent that the quark spin transfer density is dominated by the valence contribution this gives a straightforward measure of the amount of polarization of the antiquarks. Figure 1 shows our model estimates for the Drell-Yan asymmetry as a function of the invariant mass squared. These measurements are sensitive to our sea parameters and will therefore allow us to constrain the $\mathrm{SU}_{3}$ breaking $(\epsilon)$ as well as the $\bar{q}$ enhancement parameier $(c)$.

The production of jets and hadrons at large transverse momentum using polarized beams and targets presents a wide range of experimental opportunities. ${ }^{12}$ The fundamental asymmetries for the $2 \rightarrow 2$ processes are known from perturbation theory ${ }^{1}$ and can be used to determine the polarized distributions. We consider the integrated hard-scattering process $\Delta \sigma_{L}^{\text {et }}\left(p p ; p_{T}, \sqrt{s}\right)$, where $p_{T}$ is the momentum cutoff for defining a jet. At large CM energies, jet production is dorilinated by gluon-gluon scattering processes. Our explicit calculations show that a measurement of jet production with polarized beam and target would be sensitive to the gluon polarization. In figures 2 and 3 we show estimates for the polarized jet cross section, $\Delta \sigma_{L}^{\text {jet }}\left(p p ; p_{T}, \sqrt{s}\right)$ both as a function of $\sqrt{s}$ for $p_{T}=5 \mathrm{GeV}$ and as a function of $p_{T}$ at $\sqrt{s}=75 \mathrm{GeV}$. The predictions are very sensitive to the gluon polarization and so a measurement of $\Delta \sigma_{L}^{\text {jet }}$ should provide important early clues to the magnitude of $\Delta G\left(x, Q_{0}^{2}\right)$. Figure 4 shows the asymmetry $A_{L L}$ for $p p \rightarrow \pi+X$ at $\sqrt{s}=75 \mathrm{GeV}$, which is also sensitive to the polarized gluon distribution, as discussed in reference 12. These predictions shown in figures 2 through 4 can be tested with the polarized proton beam at UNK. Analysis of data at various momentum cutoffs could shed considerable light on the size of the polarized gluon distribution.

An alternate method for determining the gluon polarization is through direct photon production from a polarized beam on a polarized target. The process is expected to be 
dominated by the quark-gluon diagrams. The discussion of Berger and Qiu ${ }^{13}$ shows the importance of measurements in the small- $x$, high $p_{T}$ region where the polarized gluon distribution would be sufficiently large. The polarized proton beam at UNK could probe transverse momenta necessary to make meaningful predictions.

At this point, it seems that the immediate aim should be to do a variety of experiments to get an indication as to the size of the polarized gluon distribution and thus, the anomaly term. We should also try to gain as much information about the sea as possible. With this knowledge, we may begin to understand the constituent contributions to the spin of the proton.

\section{REFERENCES}

1. J. Qiu, G. P. Ramsey, D. Richards and D. Sivers, Phys. Rev. D39, 361 (19S9); G. P. Ramsey, Proceedings Int. Symposium on High Energy Spin Physics, K. Heller, ed., American Institute of Physics, NY, 1989, p. 786

2. P. I. Chiapetta and J. Soffer, Phys. Rev. D31, 1019 (19S5); M. Einhorn and J. Soffer, Nucl. Phys. B274, 714 (19S6).

3. R. Jaffe, Phys. Lett. 193B, 101 (1987); S. Brodsky, J. Ellis and M. Karliner Phys. Lett. 206B, 309 (1988)

4. K. Steffan, Proceedings 1988 Int. Symposium on High Energy Spin Physics, edited by K. Heller (Amer. Inst. of Physics, N.Y. 1989), p. 1093.

5. EMC Collaboration, J. Asham et al.., Phys. Lett. B206, 364 (1988)

6. B.-A. Li, et al., Univ. of Kentucky preprints 90-4 and 91-2.

7. A.V. Efremov and O.V. Teryaev, Dubna preprint E2-88-287 (1988); G. Altarelli and G. G. Ross, Phys. Lett. 212B, 391 (1988); R.D: Carlitz, J. Collins and A.H. Mueller, Phys. Lett. 214B, 229 (198S).

8. G. Altarelli and G. Parisi, Nucl. Phys. B126, 298 (1977).

9. J. D. Bjorken, Phys. Rev. 148, 467 (1966).

10. A. Gianeli, L. Nitti, G. Preparata and P. Sforza, Phys. Lett. 150B, 214 (1985) and G. Preparata, P. Ratcliffe and J. Soffer, Marseille preprint, 1989. 
11. F. Close and D. Sivers, Phys. Rev. Lett. 39, 1116 (1977); J.C.Collins, Proc. of the Workshop on Hadron Structure Functions and Parton Distributions, D. Geeseaman, et.al., eds., World Scientific, 1990.

12. G. P. Ramsey, D. Richards and D. Sivers, Phys. Rev. D37, 3140 (1988); G.P. Ramsey and D. Sivers, Phys. Rev. D43, 2861 (1991).

13. E. Berger and J.-W. Qiu, Phys. Rev. D40, 3128 (19S9). 


\section{FIGURE CAPTIONS}

1. The Drell-Yan asymmetry, $A_{L L}^{D Y}$ for $\sqrt{s}=75$ and $x_{F}=0$ plotted as a function of the invariant mass squared. The asymmetries with EMC sea distributions are represented by solid curves, where the upper curve corresponds to $(c=3)$ while the lower to $(c=1)$. The alternate model asymmetries are represented by dot-dashed curves, with the upper $(c=3)$ and lower $(c=1)$ curves, respectively.

2. The polarized jet cross section, $\Delta \sigma_{L}^{j e t}$, as a function of $\sqrt{s}$ for the momentum cutoff $p_{T}=5 \mathrm{GeV}$. The solid curve corresponds to the EMC motivated sea, and the dashed curve to the alternate sea model. The prominant curves correspond to a large gluon polarization, $\langle\Delta G\rangle=5$. The other gluon models predict a much smaller jet cross jection.

3. The polarized jet cross section, $\Delta \sigma_{L}^{j e t}$, as a function of momentum cutoff for distinguishing a jet, $p_{t}$ at $\sqrt{s}=75$. Key: solid and dashed curves near the center correspond to the large $\Delta G$ model while the curves on the left to a moderate sized $\Delta G$. The sea model designations are the same as in figure 2.

4. The asymmetry $A_{L L}$ for $p p \rightarrow \pi+X$ plotted as a function of $p_{T}$ using the distributions discussed in reference 12. Again the upper curves are predicted with the largest polarized gluon model, while the lower curves are predicted by the moderate and zero gluon models respectively, as discussed in the text. The sea distributions are designated the same as in figures 2 and 3. 


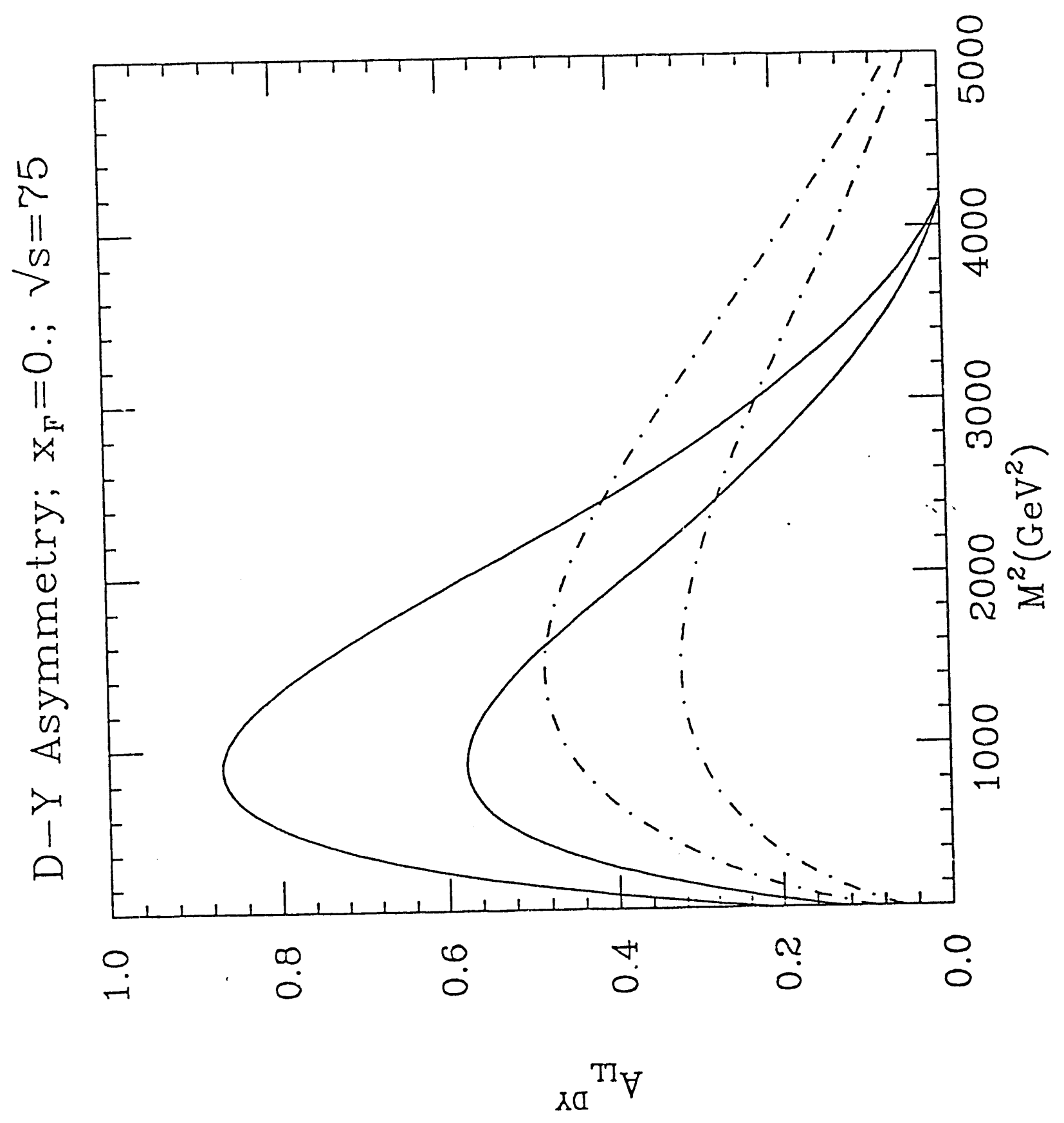




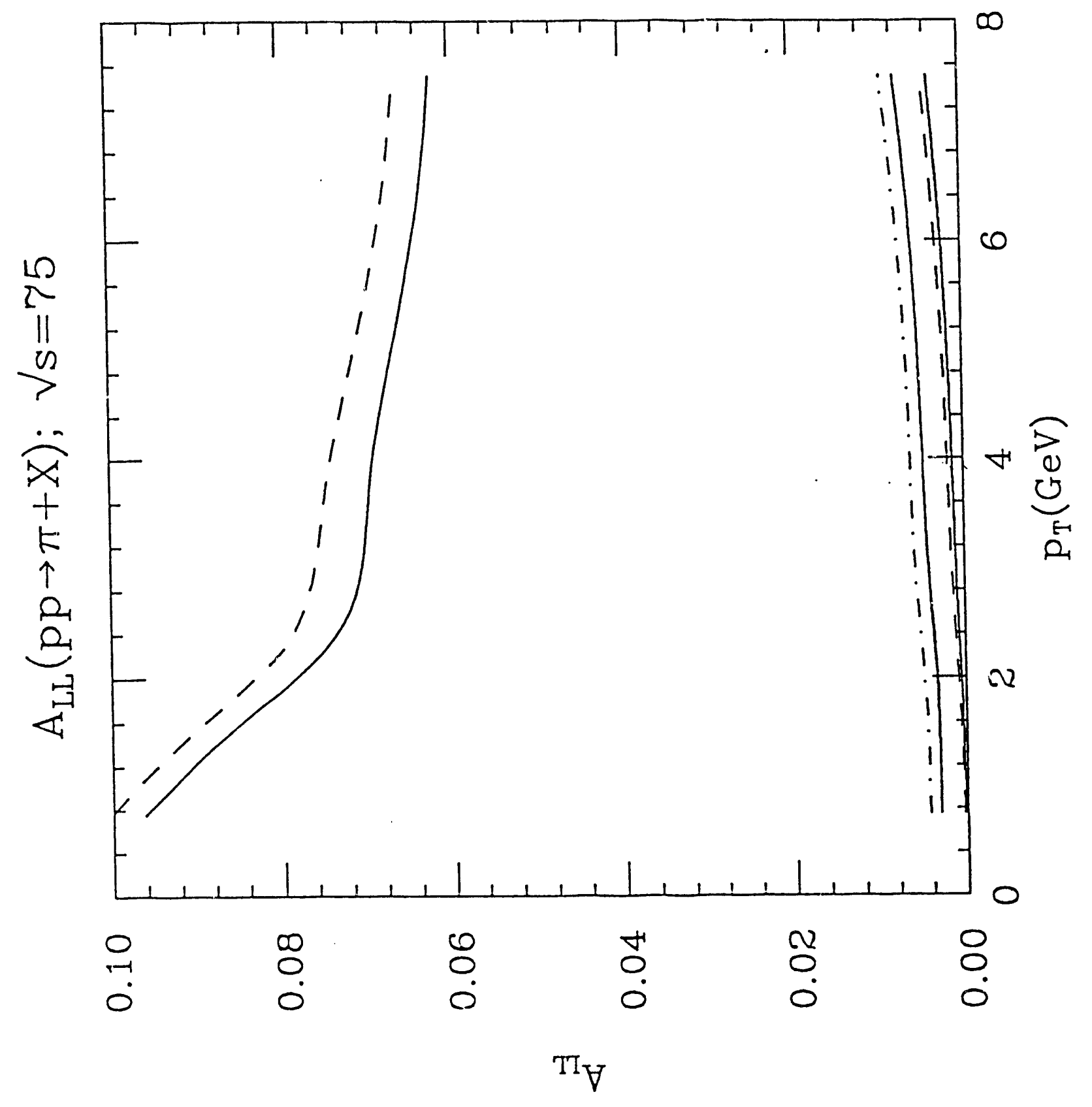




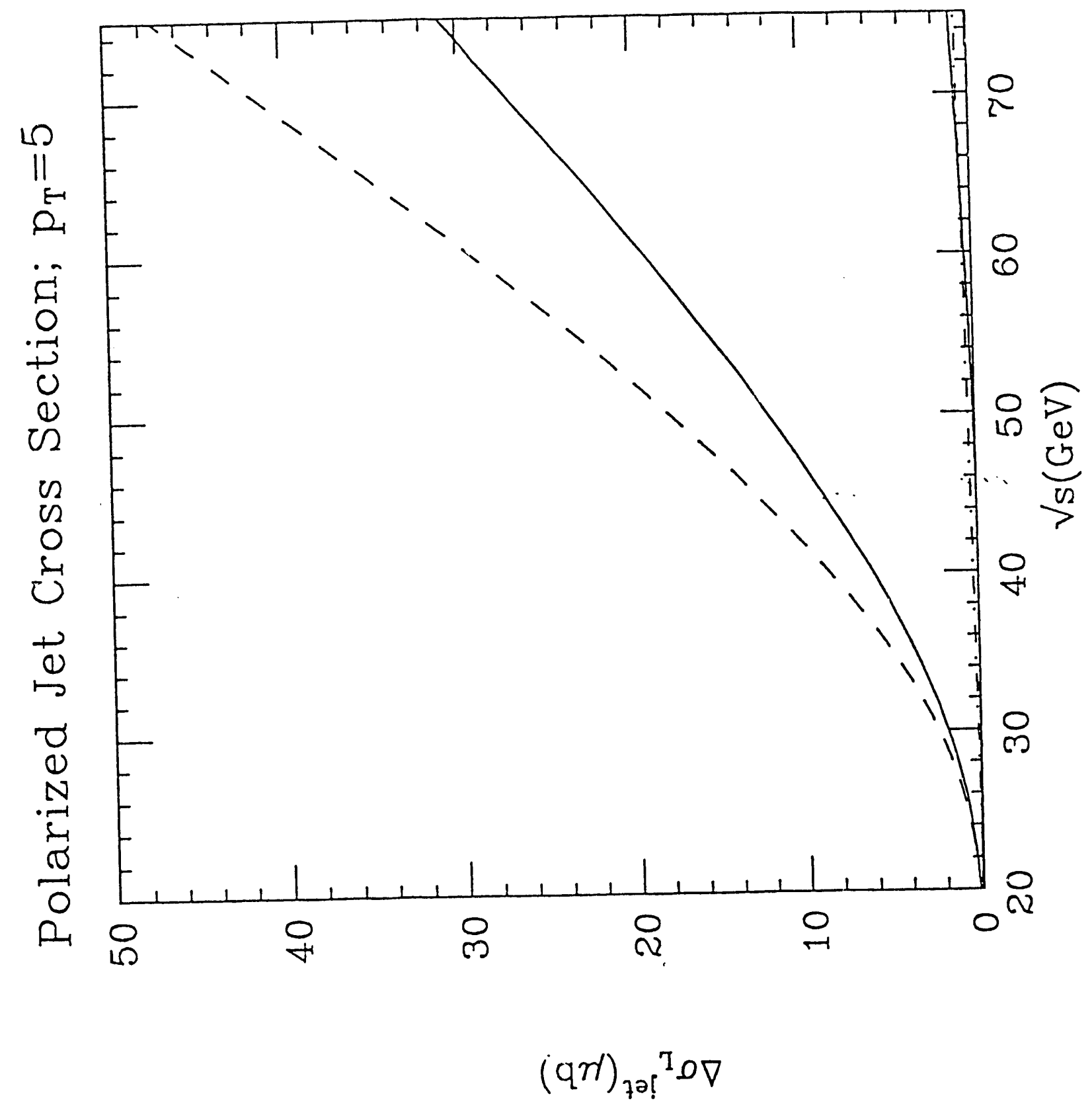




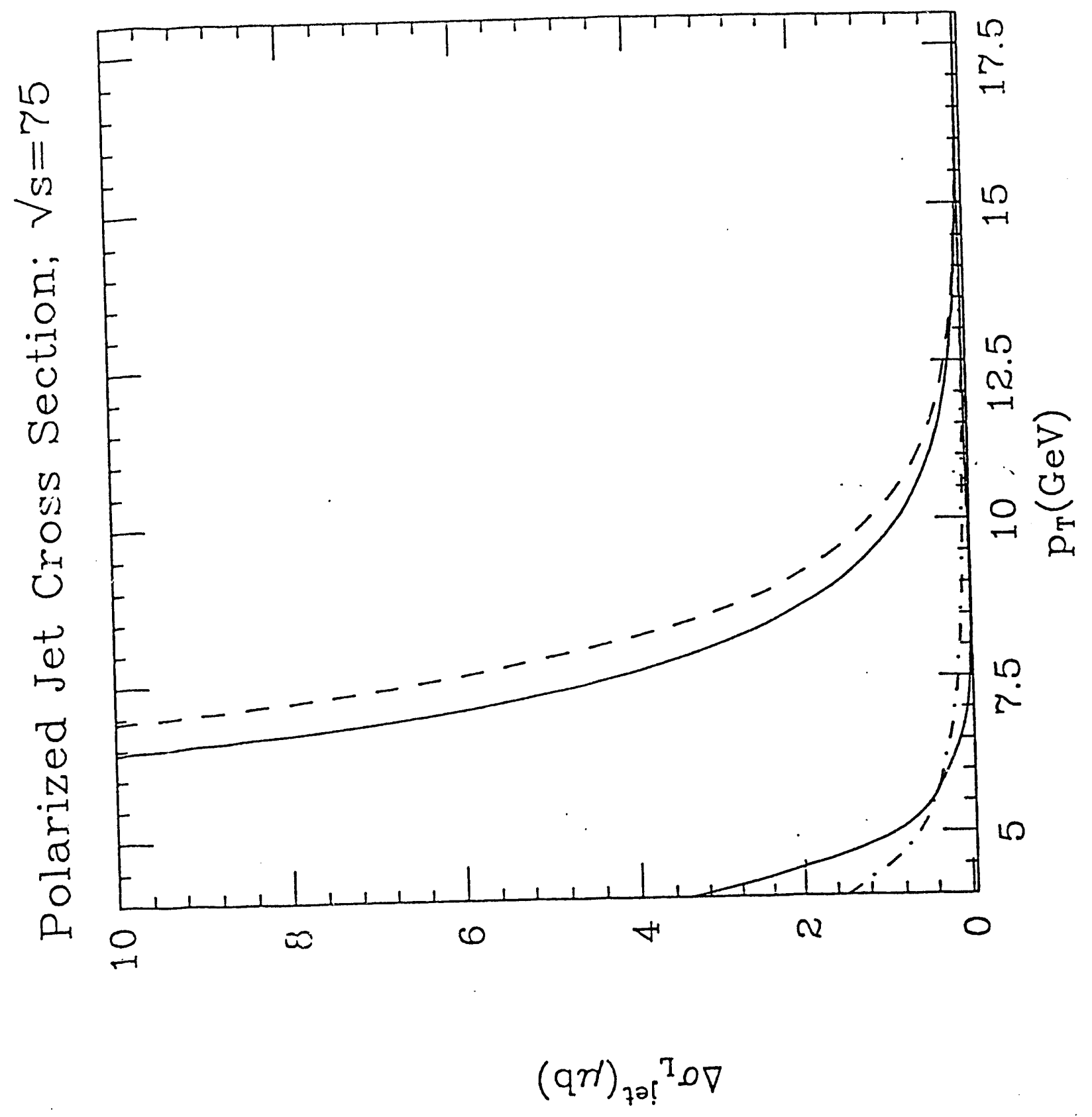


DATE

FILMED

$2 / 27 / 92$ 
